\title{
Contributions to the smut fungi of Africa. 5. First record of Thecaphora thlaspeos
}

\section{Teodor T. Denchev* \& Cvetomir M. Denchev}

Institute of Biodiversity and Ecosystem Research, Bulgarian Academy of Sciences, 2 Gagarin St., 1113 Sofia, Bulgaria

Received 29 November 2019 / Accepted 23 December 2019 / Published 25 December 2019

Denchev, T.T. \& Denchev, C.M. 2019. Contributions to the smut fungi of Africa. 5. First record of Thecaphora thlaspeos. - Mycobiota 9: 1-6. doi: 10.12664/mycobiota.2019.09.01

Abstract. Thecaphora thlaspeos, known only from Europe and East Asia (South Korea), is reported for the first time from Africa (from Algeria), on a new host plant, Arabis pubescens.

Key words: Africa, Algeria, Arabis pubescens, Brassicaceae, smut fungi, taxonomy, Thecaphora thlaspeos

\section{Introduction}

Thecaphora is a large genus in the Glomosporiaceae (Urocystidales) comprising 63 species on host plants belonging to 16 eudicot families (Vánky 2011, 2013; Kruse et al. 2018; Stajsic et al. 2018). The species of Thecaphora develop sori in various plant organs: flowers, ovaries, anthers, fruits, seeds, stems or leaves, exceptionally, in roots or tubers ( $T$. solani). Most commonly, their spores are loosely or firmly agglutinated in spore balls, composed of few to many spores (Vánky 2011, 2013). However, four species, T. australiensis, T. capensis, T. oxalidis (on Oxalidaceae), and T. thlaspeos (on Brassicaceae), produce single spores (Roets et al. 2008; Vánky 2011; Stajsic et al. 2018). The spore balls are without sterile cells, with the only exception of T. smallanthi which forms spore balls composed of spores outside and small, subhyaline sterile cells in the middle (Piepenbring 2001). In different species, the colour of the mass of spore balls or single spores is too diverse: from cinnamon brown or pale orange to dark golden or dark reddish brown. Over the past few years, various aspects of T. thlaspeos like genomics, life cycle, and plant-pathogen interactions have been intensively studied (e.g. Frantzeskakis et al. 2017; Kellner \& Göhre 2017; Courville et al. 2019).

\footnotetext{
${ }^{*}$ Corresponding author: e-mail: ttdenchev@gmail.com
} 
Thecaphora thlaspeos and five species of Urocystis are the only known smut fungi to infect hosts in the Brassicaceae (Vánky 2011). The host range of T. thlaspeos includes 16 species in Alyssum, Arabidopsis, Arabis, Cardamine, Draba, Erysimum, and Noccaea (Liro 1924; Denchev 1991; Vánky 2011). This smut fungus is recorded from Europe (Liro 1924; Ciferri 1938; Lindeberg 1959; Jørstad 1963; Vánky 1985, 1994, 2011; Denchev 1991, 2001; Zwetko \& Blanz 2004; Scholz \& Scholz 2012; Kruse et al. 2014; Frantzeskakis et al. 2017) and South Korea (Denchev et al. 2007). In this article, it is reported for the first time from Africa.

\section{Material and methods}

A dried specimen from the herbarium of Royal Botanic Garden, Madrid (MA) was examined under a light microscope (LM) and scanning electron microscope (SEM). For LM observations and measurements, spores were mounted in lactoglycerol solution ( $\mathrm{w}: \mathrm{la}$ : $\mathrm{gl}=1: 1: 2$ ) on glass slides, gently heated to boiling point to rehydrate the spores, and then cooled. The measurements of spores are given in the form: min-max (extreme values) (mean \pm 1 standard deviation). For SEM, spores were attached to specimen holders by double-sided adhesive tape and coated with gold in an ion sputter. The surface structure of spores was observed and photographed at $10 \mathrm{kV}$ accelerating voltage using a Hitachi $\mathrm{S}-3000 \mathrm{~N}$ scanning electron microscope. The description below is based entirely on the specimen examined. The shapes of spores are arranged in descending order of frequency.

\section{Taxonomy}

Thecaphora thlaspeos (Beck) Vánky, Mycotaxon 89: 111, 2004. 三 Tilletia thlaspeos Beck, Verh. K. K. Zool.-Bot. Ges. Wien 35: 362, 1886. 三 Ustilago thlaspeos (Beck) Lagerh., in Sydow, Ustilaginales Exsiccata: no. 118, 1897. E Bauhinus thlaspeos (Beck) Denchev, Mycotaxon 65: 424, 1997. 三 Tothiella thlaspeos (Beck) Vánky, Mycotaxon 70: 39, 1999. = Ustilago seminum Juel, Öfvers. Kongl. Vetensk. Akad. Förh. 51: 491, 1894. = Ustilago arabidis-alpinae Liro, Mycoth. Fenn., Fasc. 16: 108, 1939. = Ustilago cardamines Liro, Mycoth. Fenn., Fasc. 16: 108, 1939.

Figs 1-6

Infection systemic. Sori in siliquae, replacing the seeds. Spore mass powdery, light yellowish brown, released when the siliquae open. Spores single, variable in shape, subglobose, broadly ellipsoidal, ellipsoidal, globose, irregular, ovoid, reniform or elongated, $(10.5-) 11.5-17(-18.5) \times(10-) 11-13.5(-14.5)(13.7 \pm 1.3 \times 11.9 \pm 0.6) \mu \mathrm{m}\left(\mathrm{n} /{ }_{1}=100\right)$, light to medium yellowish brown; wall $0.5-1.0 \mu \mathrm{m}$ thick, echinate-verrucose, ornaments up to $0.7 \mu \mathrm{m}$ high, on a restricted area of the wall ornaments coarser and higher, up to $1.4(-$ 1.7) $\mu \mathrm{m}$ high. In SEM spore wall echinate-verrucose, punctate between the ornaments.

Specimen examined - On Arabis pubescens (Desf.) Poir.: ALGERIA, Ain Defla, Mt. Zaccar near Miliana, $36^{\circ} 18^{\prime} \mathrm{N} 02^{\circ} 17^{\prime} \mathrm{E}$, June 1920, leg. Ch. d'Alleizette, s.n. (MA 48394; the host plant as 'Arabis hirsuta DC. $\alpha$ ciliata', rev. W. Titz).

Distribution - On Brassicaceae: Alyssum reiseri Velen., Arabidopsis petraea (L.) V.I. Dorof. (Cardaminopsis petraea (L.) Hiitonen), Arabis alpina L., A. ciliata Clairv. (A. 


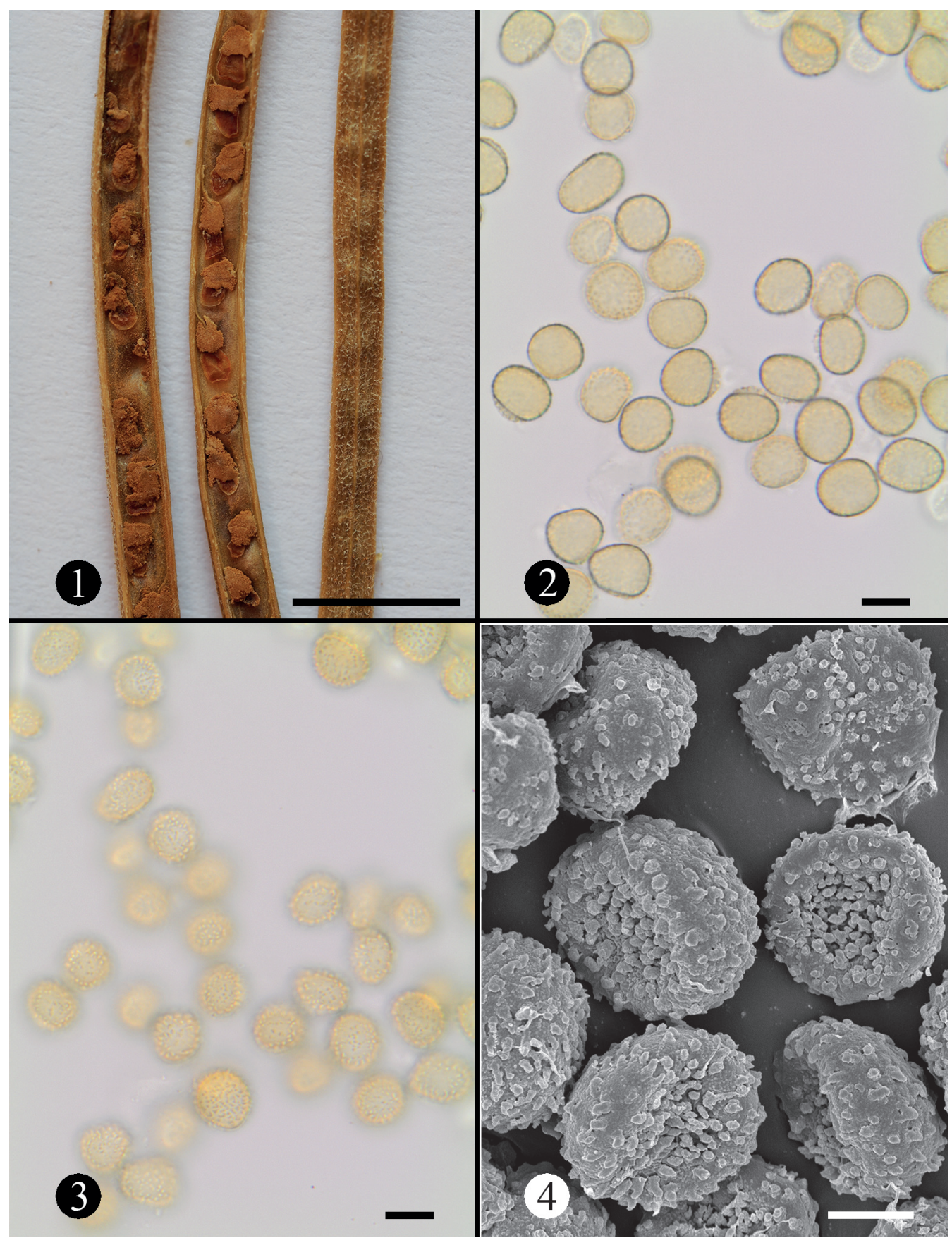

Figs 1-4. Thecaphora thlaspeos on Arabis pubescens (MA 48394). 1. Habit. 2, 3. Spores in LM (in median and surface view, respectively). 4. Spores in SEM. Scale bars: $1=0.5 \mathrm{~cm}$, $2,3=10 \mu \mathrm{m}, 4=5 \mu \mathrm{m}$ 


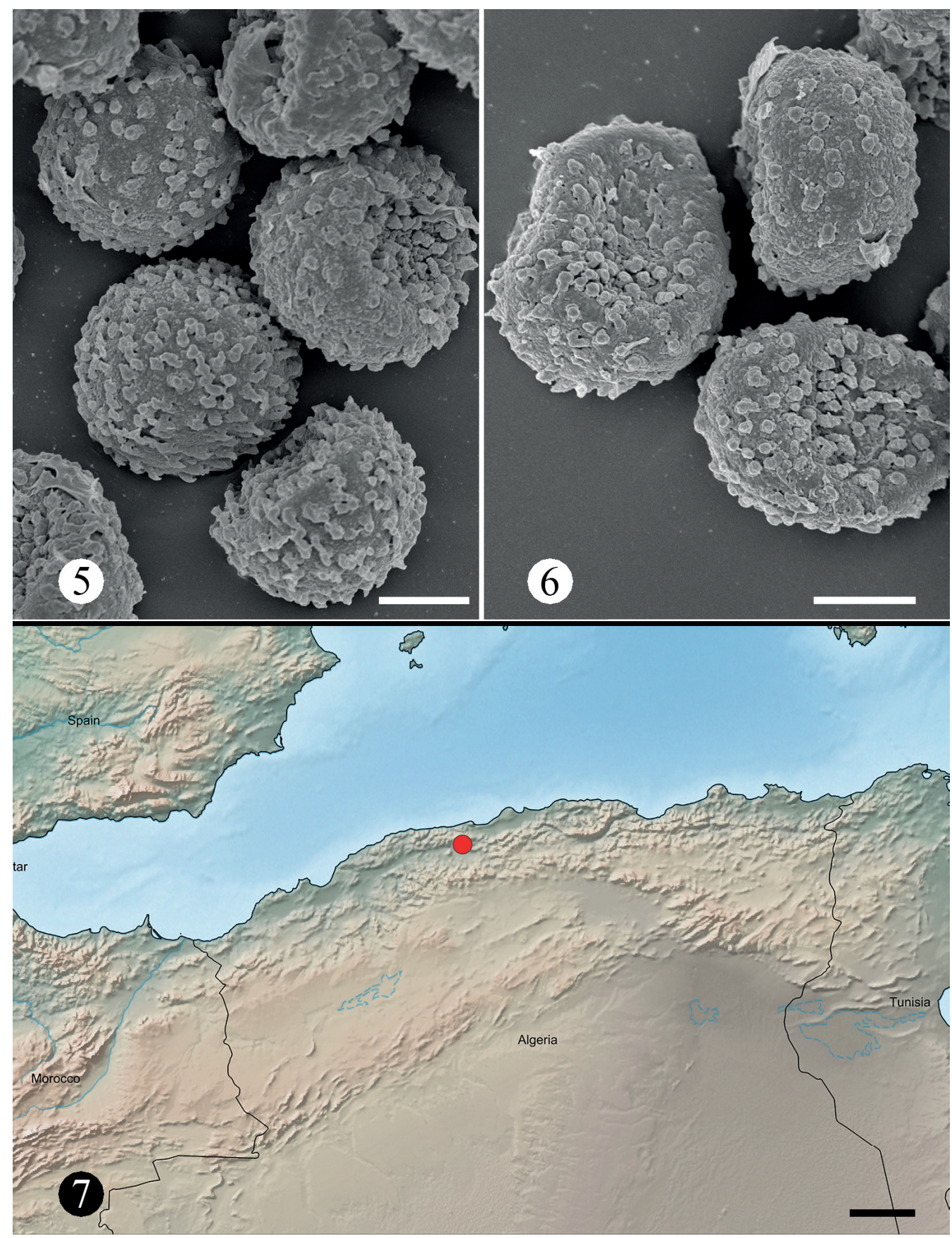

Figs 5-6. Thecaphora thlaspeos on Arabis pubescens (MA 48394) - spores in SEM. Fig. 7. Geographic distribution of Thecaphora thlaspeos in North Africa (generated with SimpleMappr, Shorthouse 2010). Scale bars: 5, $6=5 \mu \mathrm{m}, 7=500 \mathrm{~km}$ 
corymbiflora Vest), A. hirsuta (L.) Scop., A. pubescens, A. sagittata (Bertol.) DC., A. serrata var. hallaisanensis (Nakai) Ohwi, Cardamine bellidifolia L., Draba aizoides L., D. alpina L., D. incana L., Erysimum diffusum Ehrh., E. welcevii Urum., Noccaea alpestris (Jacq.) Kerguélen (Thlaspi alpinum Crantz), N. brachypetala (Jord.) F.K. Mey. (Thlaspi brachypetalum Jord.), and N. caerulescens (J. Presl \& C. Presl) F.K. Mey. (Thlaspi alpestre L.); Europe, North Africa (Algeria, Fig. 7), and Asia (South Korea).

Thecaphora thlaspeos is a rather inconspicuous species, without obvious infection symptoms. The smut fungus can be seen once siliquae are opened and the spore mass becomes exposed.

Arabis pubescens is endemic to Mediterranean North Africa, known from Morocco, Algeria, and Tunisia (Maire 1967; Pottier-Alapetite 1979; Marhold 2011). It is a new host plant record for Thecaphora thlaspeos.

Acknowledgements. This research received support (Grant no. ES-TAF-6618) from the SYNTHESYS Project http://www.synthesys.info/ which is financed by European Community Research Infrastructure Action under the FP7 "Capacities" Program at the Real Jardín Botánico (CSIC). The assistance of Prof. Maria T. Tellería, Dr Maria P. Martín, Dr Margarita Dueñas, Dr Yolanda Ruiz-León (RJB), and the staff of MA is kindly acknowledged.

\section{References}

Ciferri, R. 1938. Ustilaginales. - Flora Italica Cryptogama, Pars I, Fungi, Fasc. 17: 1-443.

Courville, K.J., Frantzeskakis, L., Gul, S., Haeger, N., Kellner, R., Heßler, N., Day, B., Usadel, B., Gupta, Y.K., van Esse, H.P., Brachmann, A., Kemen, E., Feldbrügge, M. \& Göhre, V. 2019. Smut infection of perennial hosts: the genome and the transcriptome of the Brassicaceae smut fungus Thecaphora thlaspeos reveal functionally conserved and novel effectors. - New Phytologist 222: 1474-1492. https://doi.org/10.1111/nph.15692

Denchev, C.M. 1991. New records of Bulgarian smut fungi (Ustilaginales). - Sydowia 43: 15-22. Available from: https://www.zobodat.at/pdf/Sydowia_43_0015-0022.pdf.

Denchev, C.M. 2001. Class Ustomycetes (Orders Tilletiales, Ustilaginales, and Graphiolales). - In: V. Fakirova (ed.). Fungi of Bulgaria. Vol. 4. Pp. 1-286. Editio Academica 'Prof. Marin Drinov' \& Editio Pensoft, Sofia. (In Bulgarian with an English summary)

Denchev, C.M., Choi, Y.J., Lee, S.K. \& Shin, H.D. 2007. New records of smut fungi from Korea. 1. Mycotaxon 100: 67-72.

Frantzeskakis, L., Courville, K.J., Plücker, L., Kellner, R., Kruse, J., Brachmann, A., Feldbrügge, M. \& Göhre, V. 2017. The plant-dependent life cycle of Thecaphora thlaspeos: a smut fungus adapted to Brassicaceae. - Molecular Plant-Microbe Interactions 30: 271-282. https://doi.org/10.1094/MPMI08-16-0164-R

Jørstad, I. 1963. Ustilaginales of Norway (exclusive of Cintractia on Carex). - Nytt Magasin for Botanikk 10: 85-130.

Kellner, R. \& Göhre, V. 2017. Thecaphora thlaspeos - ein Brandpilz spezialisiert auf Modellpflanzen. Biospektrum 23: 498-500. https://doi.org/10.1007/s12268-017-0828-y 
Kruse, J., Kummer, V. \& Thiel, H. 2014. Bemerkenswerte Funde phytoparasitischer Kleinpilze (2): Weitere Brandpilze. - Zeitschrift für Mykologie 80: 227-255.

Kruse, J., Kummer, V., Shivas, R.G. \& Thines, M. 2018. The first smut fungus, Thecaphora anthemidis sp. nov. (Glomosporiaceae), described from Anthemis (Asteraceae). - MycoKeys 41: 39-50. https://doi. org/10.3897/mycokeys.41.28454

Lindeberg, B. 1959. Ustilaginales of Sweden (exclusive of the Cintractias on Caricoideae). - Symbolae Botanicae Upsalienses 16(2):1-175.

Liro, J.I. 1924. Die Ustilagineen Finnlands I. - Annales Academiae Scientiarum Fennicae, Ser. A 17(1): $1-636$.

Maire, R. 1967. Flore de l'Afrique du Nord. Vol. 13. Ed. Paul Lechevalier, Paris.

Marhold, K. 2011. Brassicaceae. - In: Euro+Med Plantbase - the information resource for EuroMediterranean plant diversity. Available from: http://ww2.bgbm.org/EuroPlusMed/PTaxonDetail. asp?NameCache=Arabis\%20pubescens\&PTRefFk=7200000 (accessed 18 November 2019).

Piepenbring, M. 2001. New species of smut fungi from the neotropics. - Mycological Research 105: 757-767. https://doi.org/10.1017/S0953756200004135

Pottier-Alapetite, G. 1979. Flore de la Tunisie. Angiospermes-Dicotyledones, Apétales-Dialypétales. Ministère de l'Enseignement Supérieur et de la Recherche Scientifique et le Ministère de l'Agriculture, Tunis, Tunisia.

Roets, F., Dreyer, L.L., Wingfield, M.J. \& Begerow, D. 2008. Thecaphora capensis sp. nov., an unusual new anther smut on Oxalis in South Africa. - Persoonia 21: 147-152. https://doi. org/10.3767/003158508X387462

Scholz, H \& Scholz, I. 2012. Die Brandpilze Deutschlands, 3. Nachtrag. - Verhandlungen des Botanischen Vereins von Berlin und Brandenburg 145: 161-217.

Shorthouse, D.P. 2010. SimpleMappr, an online tool to produce publication-quality point maps. Available from: http://www.simplemappr.net (accessed 22 October 2019).

Stajsic, V., Tan, Y.P., Edwards, J. \& Shivas, R.G. 2018. Thecaphora australiensis Stajsic, Y.P. Tan \& R.G. Shivas, sp. nov. - In: P.W. Crous et al. (eds). Fungal Planet description sheets: 716-784. - Persoonia 40: 326-327. https://doi.org/10.3767/persoonia.2018.40.10

Vánky, K. 1985. Carpathian Ustilaginales. - Symbolae Botanicae Upsalienses 24(2): 1-309.

Vánky, K. 1994. European smut fungi. Gustav Fischer Verlag, Stuttgart, Jena, New York.

Vánky, K. 2011['2012']. Smut fungi of the world. APS Press, St. Paul, Minnesota, USA.

Vánky, K. 2013. Illustrated genera of smut fungi. $3^{\text {rd }}$ edn. American Phytopathological Society Press, St. Paul, Minnesota, USA.

Zwetko, P. \& Blanz, P. 2004. Die Brandpilze Österreichs. Doassansiales, Entorrhizales, Entylomatales, Georgefischeriales, Microbotryales, Tilletiales, Urocystales, Ustilaginales. - In: F. Ehrendorfer (ed.). Catalogus Florae Austriae 3(3), Biosystematics and Ecology Series 21. Verlag der Österreichischen Akademie der Wissenschaften, Wien. 